\title{
A BOUND ON THE RANK OF PURELY SIMPLE SYSTEMS
}

\author{
BY
}

FRANK OKOH

\begin{abstract}
A pair of complex vector spaces $(V, W)$ is called a system if and only if there is a C-bilinear map from $\mathrm{C}^{2} \times V$ to $W$. The category of systems contains subcategories equivalent to the category of modules over the ring of complex polynomials. Many concepts in the latter generalize to the category of systems. In this paper the pure projective systems are characterized and a bound on the rank of purely simple systems is obtained.
\end{abstract}

Introduction. The classification of a pair of linear maps $f_{1}, V_{1} \rightarrow V_{2}, f_{2}$ : $V_{1} \rightarrow V_{2}$ is connected with the graph

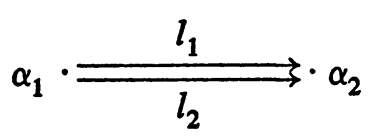

A representation of (1) assigns complex vector spaces $V_{1}, V_{2}$ to $\alpha_{1}$ and $\alpha_{2}$ respectively and linear maps $f_{1}, f_{2}$ as above to $l_{1}$ and $l_{2}$ respectively. If one restricts oneself to finite-dimensional vector spaces, then a theorem of Kronecker and Weierstrass says that every representation is a direct sum of indecomposable representations. Using this result and theorems proved by Aronszajn and Fixman we give, in \$2, a characterisation of representations that are direct sums of finite-dimensional indecomposable representations in the case when the vector spaces are of arbitrary dimension. Aronszajn became interested in these representations because of his investigation of finite-dimensional perturbations of spectral problems. He and Fixman in [1] call a representation of (1) a system. They prove results analogous to those in the theory of abelian groups. Our characterisation of pure projective systems in $\$ 2$, for instance, is analogous to the characterisation of pure projective abelian groups, with finite-dimensional indecomposable representations playing the role of cyclic groups. Our proof like the proofs of many of the results in [1] relies heavily on the structure of finite-dimensional systems and is not valid in the category of the representations of a graph like (1) with more than

Received by the editors February 25, 1976.

AMS (MOS) subject classifications (1970). Primary 15A21, 16A14, 16A46; Secondary 18G25, $20-00$.

Key words and phrases. Torsion-free system, torsion-closure, pure projective, purely simple, basis with respect to generation, rank. 
two arrows. Irrespective of the number of arrows, the category is equivalent to the category of right modules over a hereditary subring of $M_{n+1}(\mathbf{C})$, where $n$ is the number of arrows; see [6]. One wonders about the hereditary rings for which theorems analogous to those we have for systems can be proved.

$\$ 3$ is a contrast to $\$ 2$. In the category of modules over a principal ideal domain it is easily shown that there is no purely simple module of rank greater than one. Already in [4], there is an example of a purely simple system of rank two. We show that for any positive integer $n$ there exists a purely simple system of rank $n$. In $\$ 4$ it is proved that a system of rank greater than the cardinality of the continuum cannot be purely simple, i.e., has a proper pure subsystem. I do not know if there exists a purely simple system of any infinite rank. For convenience we have also included in \$1, without proof, some results and definitions in the literature that we use constantly.

We should add that (1) is a quiver as defined in [10]. As a result of new striking results interest has grown in the finite-dimensional representations of quivers and their generalizations. We refer in particular to Gabriel's results in [9]. Dlab and Ringel, using the functors introduced in [2] by Bernštein, Gel'fand and Ponomarev, have extended Gabriel's theorems to valued graphs. For details, see [3]. There is a survey in [10] of other developments in the representation of quivers and the connection with the representation of algebras. The results of our paper and the theorems in [11] can be viewed as results on the infinite-dimensional complex representations of the algebra corresponding to the quiver (1). In [12], Ringel considers the problem of obtaining an infinite-dimensional indecomposable module from finite-dimensional ones. Applying his results to systems one gets only indecomposable systems of rank $<1$. However the correspondence between nonsingular systems and modules over the complex polynomials (see [1]) enables one to get indecomposable systems of any rank less than the first inaccessible cardinal; see $[8$, Vol. II]. Such systems are not purely simple.

1. Preliminaries. All vector spaces are over the complex numbers.

DEFINITION 1.1. (a) $A$ system is a pair of vector spaces $(V, W)$ together with a system operation which is a C-bilinear map $(e, v) \mapsto e v$ of $\mathbf{C}^{2} \times V$ into $W . V$ is called the domain space and $W$ the range space. The dimension of a system $(V, W)$ is defined as $\operatorname{dim} V+\operatorname{dim} W$, where $\operatorname{dim}$ is the vector space dimension.

(b) $A$ system $(S, T)$ is a subsystem of a system $(X, Y)$ in case $S$ and $T$ are subspaces of $X$ and $Y$ respectively and es $\in T$ for all $s \in S$ and $e \in \mathbf{C}^{2}$.

(c) A homomorphism from a system $(S, T)$ to a system $(X, Y)$ is a pair of linear maps $f_{1}: S \rightarrow X, f_{2}: T \rightarrow Y$ such that $e \cdot f_{1}(s)=f_{2}(e \cdot s)$ for all $s \in S$ and $e \in \mathrm{C}^{2}$.

(d) If $(S, T)$ is a subsystem of $(X, Y)$ the quotient $(X, Y) /(S, T)$ is the system given by the pair of spaces $(X / S, Y / T)$ and the system operation is $(e$, 
$x+S) \mapsto e x+T$ for all $x \in X, e \in \mathbf{C}^{2}$.

(e) $A$ subsystem $(S, T)$ of $(X, Y)$ is a direct summand of $(X, Y)$ in case there exists a subsystem $(U, Z)$ of $(X, Y)$ such that $X=S+U$, and $Y=T+Z$.

Definition 1.2. (a) $A$ subsystem $(S, T)$ of $(X, Y)$ is said to be pure in $(X, Y)$ provided that for every intermediate subsystem $(U, Z),(S, T) \subset(U, Z) \subset(X$, $Y)$ such that $(U, Z) /(S, T)$ is finite-dimensional, $(S, T)$ is a direct summand of $(U, Z)$.

(b) A system $(X, Y)$ is said to be purely simple if it has no proper pure subsystem.

(c) A system $(V, W)$ is said to be pure-projective if it has the projective property relative to the class of pure-exact sequences.

REMARK 1.3. One can deduce from 1.2(a) that a direct sum of finite-dimensional systems is pure-projective.

Definition 1.4. (a) A system $(V, W)$ is said to be torsion-free if and only if for every $0 \neq e \in \mathbf{C}^{2}$, the map $v \mapsto e v$ is injective.

(b) Let $X, Y$ be subsets of $V, W$ resp.; there exists a smallest subsystem, $\left(V^{1}\right.$, $\left.W^{1}\right)$ of $(V, W)$ with $X \subset V^{1}, Y \subset W^{1}$ such that $(V, W) /\left(V^{1}, W^{1}\right)$ is torsion-free. $\left(V^{1}, W^{1}\right)$ is called the torsion-closure of $(X, Y)$ in $(V, W)$ or the subsystem of $(V, W)$ generated by $(X, Y)$. A subsystem $(X, Y)$ of $(V, W)$ is said to be torsion-closed in $(V, W)$ if $(X, Y)=$ torsion-closure of $(X, Y)$ in $(V$, $W)$ denoted by $\mathrm{tc}_{(V, W)}(X, Y)$.

(c) A system $(V, W)$ is said to be of rank $t$, not necessarily finite, if ( $V$, $W)=\operatorname{tc}_{(V, W)}\left(\varnothing,\left\{w_{i}\right\}_{i \in I}\right)$ and $\operatorname{card}(I)=t$ but $(V, W) \neq \operatorname{tc}\left(\varnothing,\left\{w_{j}\right\}_{j \in J}\right)$ for any $J \subsetneq I$. The set $\left\{w_{i}\right\}_{i \in I}$ is then said to be a basis of $(V, W)$ with respect to generation. We refer to [4] for the fact that this is well defined and for the proof of the theorem below.

TheOREM 1.5 [4, Theorem 2.4]. For every torsion-closed subsystem $(X, Y)$ of $(V, W), \operatorname{rank}(V, W)=\operatorname{rank}(V, W) /(X, Y)+\operatorname{rank}(X, Y)$.

Definition 1.6. Let $(V, W)$ be a system and let $w \in W$. Consider a basis ( $a$, b) of $\mathbf{C}^{2}$. We define the height $H(w)_{\theta}$ at $\theta$ relative to $(V, W)$ as follows: if the equation $b_{\theta} v=w(\theta \in \tilde{C}=\mathbf{C} \cup\{\infty\})$ and the ordered pair

$$
\left(b_{\theta}, a\right)= \begin{cases}(b-\theta a, a) & \text { if } \theta \neq \infty, \\ (a, b) & \text { if } \theta=\infty,\end{cases}
$$

has no solution in $(V, W)$ put $H^{(V, W)}(w)_{\theta}=0$. If it does consider the positive integers $k$ for which there exists a chain $\left(\left(v_{1}, v_{2}, \ldots, v_{k}\right),\left(w_{1}, w_{2}, \ldots, w_{k+1}\right)\right)$ with $b_{\theta} v_{1}=w_{1}=w, a v_{i}=w_{i+1}=b_{\theta} v_{i+1}, i=1, \ldots, k-1, a v_{k}=w_{k+1}$. Take $H^{(V, W)}(w)_{\theta}$ to be the supremum of these $k$ 's.

REMARK. (a) If $(V, W)$ is torsion-free and $0 \neq w \in W$, the chain above is unique and if $k=H^{(V, W)}(w)_{\theta}$, we call the subsystem of $(V, W)$ spanned by 
$\left(\left(v_{1}, \ldots, v_{k}\right),\left(w_{1}, \ldots, w_{k}, w_{k+1}\right)\right)$ the $\theta$-closure of $(\varnothing, w)$ in $(V, W)$. The $\left\{v_{i}\right\}_{i=1}^{k}$ and $\left\{w_{j}\right\}_{j=1}^{k+1}$ are each linearly independent. The latter fact follows from Theorem 1.7 below.

(b) A height function, $H$, is a function from $\tilde{\mathbf{C}}$ to the positive integers $\cup\{\infty\}$.

Two height functions $H$ and $H^{\prime}$ are said to be equivalent if and only if

(i) the set $\Delta=\left\{\theta \in \tilde{\mathbf{C}}: H_{\theta} \neq H_{\theta}^{\prime}\right\}$ is finite and $H_{\theta} \neq \infty \neq H_{\theta}^{\prime}$ for $\theta \in \Delta$.

(ii) If one of the functions does not assume the value $\infty$ then $\Sigma_{\theta \in \Delta} H_{\theta}=$ $\Sigma_{\theta \in \Delta} H_{\theta}^{\prime}$.

Let $\mathbf{C}(\xi)$ be the complex rational functions. $(\mathbf{C}(\xi), \mathbf{C}(\xi))_{a, b}$ is made into a system as follows: $a f=f, b f=\xi f, f \in \mathbf{C}(\xi)$. The isomorphism type of such a system is independent of choice of basis of $C^{2}$ and is denoted by $\Re[1, p$. 327].

TheOREM 1.7 [4, TheOReM 3.4]. (a) There is a one-one correspondence between equivalence classes of height functions and isomorphism types of rank 1 torsion-free systems. Given a height function $H$ it corresponds to a system $(S, T)_{a, b}^{H}$ contained in $(\mathbf{C}(\xi), \mathbf{C}(\xi))_{a, b}$ with

$$
\begin{aligned}
& S=\left[\left\{(\xi-\theta)^{-k}: 0<k<H_{\theta}+1, \theta \neq \infty\right\}\right]+\left[\left\{\xi^{k}: 0<k<H_{\infty}\right\}\right], \\
& T=\left[\left\{(\xi-\theta)^{-k}: 0 \leqslant k<H_{\theta}+1\right\}\right]
\end{aligned}
$$

where $(\xi-\theta)^{-k}$ denotes $\xi^{k}$ when $\theta=\infty$ ( $k$ is an integer) $(S, T)_{a, b}^{H}$ is torsion-free and of rank 1 .

(b) If $(V, W)$ is of rank 1 then the height functions corresponding to the heights of nonzero elements at $\boldsymbol{\theta} \in \tilde{\mathrm{C}}$ are all equivalent.

We note as in [4] that equivalence of height functions is independent of the choice of basis of $\mathbf{C}^{2}$.

Let $H$ be the height function defined by $H_{\theta}=0$ for $\theta \in \mathrm{C}$ and $H_{\infty}=\infty$. The domain space and range space of $(S, T)_{a, b}^{H}$ are respectively the space of polynomials $\mathrm{C}[\xi]$. The equivalence class of $H$, and therefore the isomorphism type of $(S, T)_{a, b}^{H}$, depends on $\dot{a}=\{\alpha a: \alpha \in \mathbf{C}\}$. We shall denote this isomorphism type by $\mathscr{P}_{\dot{a}}$. The isomorphism type of finite-dimensional torsion-free rank 1 systems will be denoted by III $^{m}$, where $m-1$ is the dimension of the domain space and $m$ the dimension of the range space. A system of type III $^{m}$ may be assumed to be spanned by a chain $\left(\left(x_{1}, \ldots, x_{m-1}\right),\left(y_{1}, \ldots, y_{m}\right)\right)$ with $a x_{1}=y_{1}, a x_{i+1}=y_{i+1}=b x_{i}, i=$ $1, \ldots, m-2, b x_{m-1}=y_{m}$.

THEOREM 1.8. Let $E: 0 \rightarrow(X, Y) \rightarrow(U, Z) \rightarrow(V, W) \rightarrow 0$ be an exact sequence of systems such that $(X, Y)=\Sigma_{j \in J} \cdot\left(X_{j}, Y_{j}\right), J$ finite. Suppose each $\left(X_{j}, Y_{j}\right)$ is of type $\mathrm{III}^{m_{j}}$ spanned by $\left(\left(x_{1 j}, \ldots, x_{m_{j}-1, j}\right),\left(y_{1 j}, \ldots, y_{m_{j},}\right)\right)$ and $(V$, $W)$ is torsion-free. Then $E$ is congruent to an extension in which the middle 
term is $(X \oplus V, Y \oplus W)$ with the system operation defined by

$$
\begin{aligned}
& a\left(\sum_{j \in J} \alpha_{j} x_{j}, v\right)=\left(a \sum \alpha_{j} x_{j}+\sum l_{j}(v) y_{1 j}, a v\right), \\
& b\left(\sum_{j \in J} \alpha_{j} x_{j}, v\right)=\left(b \sum \alpha_{j} x_{j}, b v\right), \quad \alpha_{j} \in \mathbf{C},
\end{aligned}
$$

where $l_{j}$ are linear functionals on $V$. The system operations on the first and the second components on the right-hand side are in $(X, Y)$ and $(V, W)$ respectively.

Proof. In the case when $\operatorname{card}(J)=1$, this theorem is proved in [11, Theorem 5.3] and the remark following it. The proof there generalizes to any $J$ with $\operatorname{card}(J)<\infty$.

Even if $(X, Y)$ in 1.8 is not finite-dimensional we still have

LEMMA $1.9([11, \S 4]$ OR [5]). Let $E: 0 \rightarrow(X, Y) \rightarrow(U, Z) \rightarrow(V, W) \rightarrow 0$ be an exact sequence of systems. Then $E$ is congruent to an extension in which the middle term is $(X \oplus V, Y \oplus W)$ and the system operation is given by

$$
a(x, v)=(a x+a * v, a v), \quad b(x, v)=(b x+b * v, b v),
$$

where * is a C-bilinear map from $V$ to $Y$.

LEMMA 1.10. Let $(U, Z)$ be a torsion-free system of rank $n$ and $\left(X_{0}, Y_{0}\right) a$ finite-dimensional torsion-closed subsystem of rank $n-1$, such that $(U, Z) /\left(X_{0}, Y_{0}\right)$ is of type $\mathscr{P}_{\dot{a}}$. If $(X, Y)$ is any other finite-dimensional torsion closed subsystem of $(U, Z)$ of rank $n-1$, then $(U, Z) /(X, Y)$ is also of type $\mathscr{P}_{\dot{a}}$.

Proof. We may by Lemma 1.9 and the definition of $\mathscr{P}_{\dot{a}}$ take $U=X_{0} \oplus$ $V_{1}, Z=Y_{0} \oplus W_{1}$ with $\left(V_{1}, W_{1}\right)$ the system $(\mathbf{C}[\xi], \mathbf{C}[\xi])_{a, b} \subset(\mathbf{C}(\xi), \mathbf{C}(\xi))_{a, b}$. The system operation in $(U, Z)$ is as in 1.9. For $u=\left(x_{0}, f\right) \in U\left(z=\left(y_{0}\right.\right.$, $f) \in Z$ respectively) we call $f$ the polynomial part of $u$ ( $z$ respectively). Suppose $(X, Y)$ is a torsion-closed subsystem of $(U, Z)$ of rank $n-1$. By Theorem 1.5, $(V, W)=(U, Z) /(X, Y)$ is of rank 1 and it is infinite-dimensional by the hypothesis. To prove that $(V, W)$ is of type $\mathscr{P}_{\dot{a}}$ it is therefore enough by 1.7 to show that, for a nonzero element $w$ in $W$,

$$
\begin{array}{ll}
H^{(V, W)}(w)_{\theta}=0 & \text { for almost all } \theta \in \mathbf{C}, \\
H^{(V, W)}(w)_{\theta}<\infty & \text { for all } \theta \in \mathbf{C} .
\end{array}
$$

This is equivalent to showing (again, by 1.7) that the following subsystem of $(V, W)$

$$
\left(V^{0}, W^{0}\right)=\left[\sum_{\theta \in \mathrm{C}}\{\theta \text {-closure of } w \text { in }(V, W)\}\right]
$$

is finite-dimensional. 
To prove (2) we shall need the above representation of $U$ and $Z$ and

(3) Let $S$ be a subset of $\mathrm{C}[\xi]$. Then $S$ generates a finite-dimensional subspace of $\mathrm{C}[\xi]$ if and only if there exists a positive integer $N$ such that, for every $s \in S$, degree of $s \leqslant N$. We say, in that case, that $S$ has bounded degree.

If $\left(y_{0}, f\right) \in Z$, let $\overline{\left(y_{0}, f\right)}$ denote the image of $\left(y_{0}, f\right)$ under the natural projection on $W=Z / Y$. From Theorem 1.8 and the definition of the system operation on the quotient $(V, W) /(X, Y)$,

(4) If $\overline{\left(y_{0}, f\right)} \neq 0$ then $H^{(V, W)}\left(\left(y_{0}, f\right)\right)_{\theta}>0$ implies that $\xi-\theta$ is a zero of $f$ modulo the polynomial parts of $Y$.

Let $V_{p}^{0}\left(W_{p}^{0}\right)$ denote the polynomial parts in $\{u+x: x \in X, u+X \in$ $\left.V^{0}\right\},\left(\left\{z+y: y \in Y, z+Y \in W^{0}\right\}\right)$. Since $(X, Y)$ is finite-dimensional (4) and (3) imply that $V_{p}^{0}, W_{p}^{0}$ are both of bounded degree. Hence, by (3), [ $\left.V_{p}^{0}\right]$, $\left[W_{p}^{0}\right]$ are finite-dimensional vector spaces. It is readily seen that

$$
\left(V^{0}, W^{0}\right) \subset \overline{\left(\left[V_{p}^{0}\right], \mathbf{C}^{2}\left[V_{p}^{0}\right]\right)}+\overline{\left(X_{0}, Y_{0}\right)}+\overline{\left(0,\left[W_{p}^{0}\right]\right)} \subset(V, W)
$$

where

$$
\begin{aligned}
\mathbf{C}^{2}\left[V_{p}^{0}\right] & =\left\{e v: e \in \mathbf{C}^{2}, v \in\left[V_{p}^{0}\right]\right\} \text { and } \\
\overline{\left(X_{0}, Y_{0}\right)} & =\left(\left(X_{0}, Y_{0}\right)+(X, Y)\right) /(X, Y) .
\end{aligned}
$$

$\overline{\left(\left[V_{p}^{0}\right], \mathbf{C}^{2}\left[V_{p}^{0}\right]\right)}$ is similarly defined. Therefore $\left(V^{0}, W^{0}\right)$ is finite-dimensional as required.

LeMma 1.11. Let $E: 0 \rightarrow(X, Y) \rightarrow(U, Z) \rightarrow(V, W) \rightarrow 0$ be an exact sequence of systems where $(X, Y)$ is torsion-free of rank $n$ and finitedimensional and $(V, W)$ is of type $\mathscr{P}_{\dot{a}}$. Then $(U, Z)$ is also an extension of $a$ system $\left(X_{0}, Y_{0}\right)$ of type $\bigoplus_{n} \mathrm{III}^{1}$ by a system of type $\mathscr{P}_{\dot{a}}$.

Proof. By the preceding Lemma and Theorem 1.5 it is sufficient to find a subsystem $\left(X_{0}, Y_{0}\right) \subset(U, Z)$ that is torsion-closed and of type $\bigoplus_{n} \mathrm{III}^{1}$. Using Theorem 1.7 we may take $(V, W)$ to be the system

$$
(\mathbf{C}[\xi], \mathbf{C}[\xi])_{a, b} \subset(\mathbf{C}(\xi), \mathbf{C}(\xi))_{a, b}
$$

By [1, Theorem 4.3], $(X, Y)$ is of type $\bigoplus_{j=1}^{n} \mathrm{III}^{m}$. So we also assume that $(X$, $Y)$ and $(U, Z)$ are of the form given in the hypothesis and conclusion respectively of Theorem 1.8. In the notation of 1.8 let

$$
\begin{aligned}
\left(X_{0}, Y_{0}\right)= & \left(0,\left[y_{m_{1}, 1}+y_{12}\right]\right)+\left(0,\left[y_{m_{2}, 2}+y_{13}\right]\right)+\cdots \\
& +\left(0,\left[y_{m_{n-1}, n-1}+y_{1 n}\right]\right)+\left(0,\left[y_{m_{n}, n}+1\right]\right) .
\end{aligned}
$$

It is of type $\bigoplus_{n}$ III $^{1}$.

Suppose $b_{\theta}(x, v) \in Y_{0}$. Then

$$
b_{\theta}(x, v)=\gamma_{1}\left(y_{m_{1}, 1}+y_{12}\right)+\cdots+\gamma_{n}\left(y_{m_{n}, n}+1\right) ; \quad \gamma_{j} \in \mathbf{C} .
$$


Let $x=\sum_{i=1}^{m_{1}-1} \alpha_{i 1} x_{i 1}+\cdots+\sum_{i=1}^{m_{n}-1} \alpha_{i n} x_{i n}$. (If $m_{j}=1$, the $j$ th term of $x$ in the above expression is 0 .)

If $\theta \neq \infty$, we conclude from the definition of the system operation in ( $U$, $Z$ ) that $\gamma_{n} /(\xi-\theta)=v \in \mathbf{C}[\xi]$; hence $\gamma_{n}=0=v$. Applying the definition of the $b_{\theta}$-action and equating the coefficients of $y_{j k}, j=1, \ldots, m_{k}, k=$ $1, \ldots, n$, leads to

$$
\begin{aligned}
& -\theta \alpha_{1, k}=\gamma_{k-1}, \quad \gamma_{k-1}=0 \quad \text { if } k=1, \\
& \alpha_{1 k}-\theta \alpha_{2 k}=0, \\
& \alpha_{2 k}-\theta \alpha_{3 k}=0, \\
& \vdots \\
& \alpha_{m_{k}-2}-\theta \alpha_{m_{k}-1,1}=0, \\
& \alpha_{m_{k}-1,1}=\gamma_{k}, \quad k=1, \ldots, n .
\end{aligned}
$$

From (6) we deduce that $(x, v)=(0,0)$. The same conclusion is valid if $\theta=\infty$, i.e., $b_{\theta}=a$. Therefore $\left(X_{0}, Y_{0}\right)$ is torsion-closed and we are done with the proof of Lemma 1.11 .

LEMMA 1.12. Let $(X, Y)$ be a purely simple torsion-free system. Then any proper torsion-closed subsystem of $(X, Y)$ which is of finite rank is a system of type $\bigoplus_{i \in I} \mathrm{III}^{m_{1}}$, I finite.

Proof. Let $\left(X_{0}, Y_{0}\right) \neq(X, Y)$ be torsion-closed of rank $t$. If $t=1$ and $\left(X_{0}\right.$, $\left.Y_{0}\right)$ is infinite-dimensional then it is pure in $(X, Y)$ by [4, Theorem 5.2], contradicting $(X, Y)$ purely simple. So $\left(X_{0}, Y_{0}\right)$ is of type III $^{m_{1}}$. We assume the lemma for all torsion-closed $\left(X_{1}, Y_{1}\right) \subset(X, Y)$ of rank $<t-1$. Let $\left(X_{0}\right.$, $\left.Y_{0}\right)$ be a torsion-closed subsystem of $(X, Y)$ of rank $t$. If it has no direct summand of type $\mathrm{III}^{m_{1}}$, it will be pure in $(X, Y)$ by [7, Theorem 1]. So $\left(X_{0}\right.$, $\left.Y_{0}\right)$ has a direct summand of type III $^{m_{1}}$. The latter has a direct complement, $\left(X_{2}, Y_{2}\right)$ in $\left(X_{0}, Y_{0}\right)$ of rank $t-1 .\left(X_{2}, Y_{2}\right)$ is torsion-closed in $(X, Y)$ and hence by the induction hypothesis it is a system of type $\bigoplus_{t=2}^{n} \mathrm{III}^{\mathrm{m}}$.

Lemma 1.14 is readily deduced from Theorem 1.13. A real number $x=$ $a^{1 / m}$ is said to be radical if and only if $a$ and $m$ are positive integers. The exponent of a radical number is the least positive integer $m$ such that $x^{m}$ is a positive integer.

THEOREM 1.13 [13]. Let $x_{1}, \ldots, x_{n}$ be radical numbers with exponents $m_{1}, \ldots, m_{n}$. If the positive integers $x_{1}^{m_{1}}, \ldots, x_{n}^{m_{n}}$ are coprime then $x_{1}, \ldots, x_{n}$ generate a field extension of the rationals of degree $m_{1} \cdot m_{2} \cdots \cdots m_{n}$.

LEMMA 1.14. Let $P$ be the set of all positive rational primes and for $L \subset P$ let $Q_{L}$ be the field extension obtained by adjoining to $Q$ the real numbers $\sqrt[l]{n}$ for all positive rational integers $n$ and all $l \in L$. If $L_{1}$ is not contained in $L_{2}$, then 
$Q_{L_{1}}$ is not contained in $Q_{L_{2}}(\alpha), \alpha$ a complex number algebraic over $Q$.

2. Pure projective systems. We refer to [1] for a description of systems of type $\mathrm{I}^{m}, \mathrm{II}_{\theta}^{m}$ and a proof of

THEOREM 2.1 [1, THEOREM 4.3]. Every finite-dimensional system is a direct sum of finite-dimensional indecomposable subsystems, i.e., subsystems of type $\mathbf{I}^{m}$, $\mathrm{II}_{\theta}^{m}$ or $\mathrm{III}^{m}$.

In this section we shall give a characterisation of those systems that are direct sums of finite-dimensional subsystems. In a system $(V, W)=$ $\bigoplus_{i \in I}\left(V^{i}, W^{i}\right), \quad I$ an arbitrary indexing set, we have $H\left(\left(w_{i}\right)_{i \in I}\right)_{\theta}=$ $\inf \left\{H\left(w_{i}\right)_{\theta}: i \in I\right\}$. Using this and [1, Theorem 6.6] we deduce

Proposition 2.2. If $(V, W)$ is torsion-free and a direct sum of finite-dimensional indecomposable subsystems, then any subsystem of $(V, W)$ has a direct summand of type $\mathrm{III}^{m}$ for some positive integer $m$.

THEOREM 2.3. A system $(V, W)$ is pure-projective if and only if it is a direct sum of finite-dimensional indecomposable subsystems.

Proof. We have already remarked in 1.3 that a direct sum of finite-dimensional systems is pure projective.

Let $(V, W)$ be a pure projective system and let $\left(V_{\alpha}, W_{\alpha}\right)_{\alpha \in I}$ be the set of all finite-dimensional subsystems of $(V, W)$ and $\left(\kappa_{\alpha}, \lambda_{\alpha}\right)$ the inclusion map. Let

$$
(X, Y)=\sum_{\alpha \in I} \bigoplus\left(v_{\alpha}, w_{\alpha}\right), \text { and }(\kappa, \lambda)=\sum_{\alpha \in I}\left(\kappa_{\alpha}, \lambda_{\alpha}\right) .
$$

Let $(K, L)=(\operatorname{ker} \kappa, \operatorname{ker} \lambda)$. We get the exact sequence

$$
0 \rightarrow(K, L) \rightarrow(X, Y) \rightarrow(V, W) \rightarrow 0 .
$$

In the abelian category of systems, purity as defined in 1.2 is 9 -purity, where $g$ is the family of finite-dimensional systems, in the terminology of [14]. By [14, Theorem 2.4], $(K, L)$ is pure in $(X, Y)$. Since $(V, W)$ is pure projective, we get that

$$
(X, Y) \text { is isomorphic to }(K, L) \oplus(V, W) \text {. }
$$

$(X, Y)$ is a direct sum of finite-dimensional systems, so by 2.1 it is a direct sum of finite-dimensional indecomposable subsystems. Therefore $t(X, Y)$, where $t$ stands for the 'torsion part' is a direct summand of $(X, Y)$. From $(\dagger)$ we get that $t(X, Y)=t(K, L)+t(V, W)$. Therefore $t(V, W) \subset(V, W)$ is a direct summand of $(X, Y)$, hence a direct summand of $(V, W)$. Since $(V, W)$ is pure-projective so is $t(V, W)$. That $t(V, W)$ is a direct sum of finitedimensional indecomposable subsystems follows from [1, Propositions 9.16, 9.19 and p. 338] and [8, Vol. I, Theorem 30.2]. So in ( $\dagger$ ) we may assume that $(V, W)$ is torsion-free. Therefore, $(X, Y)$ is a direct sum of finite-dimensional 
subsystems of type III $^{m}$, for various positive integers $m$. The claim that $(V$, $W)$ also has the latter property is established by imitating the proof of Theorem 86.7 in [8] after first proving that direct summands of systems that are direct sums of subgroups of type III $^{m}$ are separable. See [8] for definition. The necessary modifications to the results quoted in the proof of Theorem 86.7 in [8] are immediate. This completes the proof of Theorem 2.3.

Corollary 2.4. A direct summand of a system which is a direct sum of finite-dimensional indecomposable subsystems is itself a direct sum of finite-dimensional indecomposable subsystems.

Proof. A direct summand of a pure projective system is pure projective. Therefore 2.4 follows from 2.3 .

3. Existence of purely simple systems of arbitrary finite rank.

THEOREM 3.1. For each positive integer, $n$, there exists a purely simple system of rank $n$.

Proof. Let

$$
\begin{aligned}
V & =\mathbf{C}[\xi], \\
W & =V \dot{+}\left[w_{2}\right] \oplus \cdots \oplus\left[w_{n}\right], \quad \text { where } w_{l} \neq 0 \text { for } l=2, \ldots, n .
\end{aligned}
$$

We make $(V, W)$ into a system by requiring for a fixed basis $(a, b)$ of $\mathbf{C}^{2}$ the following relations.

$$
\begin{aligned}
& a \xi^{k}=\xi^{k}+\alpha_{2 k} w_{2}+\cdots+\alpha_{n k} w_{n}, \quad k=0,1,2, \ldots, \\
& b \xi^{k}=\xi^{k+1}
\end{aligned}
$$

where $\alpha_{l k}=\sqrt[p_{1}]{k}$ and $p_{l}$ is the $(l-1)$ th prime in the usual ordering of the positive rational primes, $l=2, \ldots, n$.

We shall now show that $(V, W)$ is of rank $n$ and it is purely simple. It is straightforward to see that $(V, W)$ is torsion-free. The subsystem $(0$, $\left.\sum_{l=2}^{n} \oplus \mathbf{C} w_{l}\right)$ is a torsion-closed subsystem of $(V, W)$ of rank $n-1$. $(V, W) /\left(0, \sum_{l=2}^{n} \oplus \mathbf{C} w_{l}\right)$ is of type $\mathscr{P}_{a}$. Hence, by Theorem $1.5, \operatorname{rank}(V$, $W)=n$. To avoid repetition "pure subsystem" means a nontrivial pure subsystem. Any pure subsystem $(X, Y) \subset(V, W)$ is torsion-closed by $[4$, Lemma $2.1(\mathrm{~g})]$ and hence by $1.5, \operatorname{rank}(X, Y)<n$. We shall show by induction that $(V, W)$ has no pure subsystem.

Suppose $(X, Y)$ is a rank 1 infinite-dimensional pure subsystem of $(V, W)$. Then $(X, Y)=\operatorname{tc}(\varnothing, y), 0 \neq y \in Y$.

$$
y=\sum_{l=2}^{n} \beta_{l} w_{l}+\sum_{i=0}^{t} \gamma_{i} \xi^{i}, \quad \beta_{l}, \gamma_{i} \in \mathbf{C} .
$$

Since $(X, Y)$ is torsion-closed in $(V, W), H^{(X, Y)}\left(y_{1}\right)_{\theta}=H^{(V, W)}\left(y_{1}\right)_{\theta}$ for all 
$y_{1} \in Y$ and all $\theta \in \tilde{\mathbf{C}}$. Denote this common height by $H\left(y_{1}\right)_{\theta}$. So by Theorem 1.7

$$
\sum_{\theta \in \tilde{\mathbf{C}}} H(y)_{\theta}=\infty
$$

This implies that $y$ is not in $\Sigma_{l=2}^{n} \oplus \mathbf{C} w_{l}$ and so we may assume that $\gamma_{t} \neq 0$ in (7). The sum of the heights of $y$ at $\theta \in \mathbf{C}$ does not exceed the corresponding sum for the image of $y$ in $(V, W) /\left(0, \sum_{l=2}^{n} \oplus \mathbf{C} w_{l}\right)$ and the latter is finite by Theorem 1.7. Thus $H(y)_{\infty}=\infty$. Using the definition of the system operation in $(V, W)$ this leads to

$$
\sum_{i=0}^{t} \gamma_{i} \alpha_{l, k+i}=0, \quad k=1,2, \ldots, l=2, \ldots, n .
$$

From (8) and the assumption $\gamma_{t} \neq 0$ we conclude that for $l=2$, for instance,

$$
\sqrt{m} \in K=Q\left(\gamma_{0}, \gamma_{1}, \ldots, \gamma_{t}, \sqrt{2}, \sqrt{3}, \ldots, \sqrt{t+1}\right)
$$

for all positive integers $m$. Hence in the notation of Lemma $1.14 Q_{\{2\}} \subset K_{0} \subset$ $K$ where $K_{0}=\{k \in K: K$ is algebraic over $Q\}$. However this is not possible because $Q_{\{2\}}$ is not a finite extension of $Q$ as is immediately deduced from 1.13. Therefore $(V, W)$ does not contain a rank 1 infinite-dimensional pure subsystem. We now assume that $(V, W)$ does not contain an infinite-dimensional pure subsystem of rank $\leqslant s<n$ and show that it cannot contain one of rank $s+1$. We shall assume the contrary and proceed to get a contradiction.

Let $(X, Y)$ be an infinite-dimensional pure subsystem of rank $s+1$. Any pure subsystem of $(X, Y)$ has rank $\leqslant s$ and so by the transitivity of purity [1, Proposition 5.2(a)] and the induction hypothesis, $(X, Y)$ has no pure infinitedimensional subsystem. It has no finite-dimensional pure subsystem because such a subsystem would be a direct summand in $(X, Y)$ whose complement in $(X, Y)$ would be an infinite-dimensional pure subsystem, [1, Theorem 5.5 and Proposition 5.2(e)]. Therefore $(X, Y)$ is purely simple. Let $\left(X_{0}, Y_{0}\right)$ be a torsion-closed subsystem of $(X, Y)$ of rank $s$. By Lemma $1.12,\left(X_{0}, Y_{0}\right)$ is finite-dimensional. So $(X, Y) /\left(X_{0}, Y_{0}\right)$ is a rank 1 infinite-dimensional system. It is of type $\mathcal{P}_{\dot{a}}$ by an argument identical to the proof of (2) in Lemma 1.10. By 1.11 we may further assume that $\left(X_{0}, Y_{0}\right)$ is of type $\bigoplus_{s} \mathrm{III}^{1}$, i.e., $\left(X_{0}, Y_{0}\right)=\sum_{i-1}^{s} \cdot\left(0, \mathbf{C} y_{i}\right)$ where each $y_{i} \neq 0$. Let

$$
\begin{aligned}
y_{i} & =\sum_{j=0}^{m_{i}} \gamma_{j i} \xi^{j}+\sum_{l=2}^{n} \chi_{l i} w_{l}, \quad i=1, \ldots, s, \\
& =p_{i}+\sum_{l=2}^{n} \chi_{l i} w_{l}, \quad \gamma_{j i}, \chi_{l i} \in \mathbf{C} .
\end{aligned}
$$


Since $(X, Y)$ is purely simple, hence indecomposable, and infinite-dimensional it has subsystems of type $\mathrm{III}^{m}$ for arbitrarily large $m$, by [1, Theorem 6.6]. Using the definition of the system operation in $(V, W)$ this implies that $Y$ contains polynomials of arbitrarily large degree. As $Y_{0}$ is finite-dimensional, we may pick a generator of $(X, Y) /\left(X_{0}, Y_{0}\right), y=\sum_{j=0}^{m} \gamma_{j} \xi^{j}+Y_{0}=p$ $+Y_{0}$, where $p=\sum_{j=0}^{m} \gamma_{j} \xi^{j}$ with $\gamma_{m} \neq 0$ and $m>\max \left(m_{1}, m_{2}, \ldots, m_{s}\right)$ in (9) and $H^{(V, W)}(p)_{\infty} \neq 0$. Since $(X, Y) /\left(X_{0}, Y_{0}\right)$ is of type $\mathscr{P}_{\dot{a}}$, we conclude that

$$
H^{(X, Y) /\left(X_{0}, Y_{0}\right)}(y)_{\infty}=\infty .
$$

Using the definition of the system operation on $(X, Y) /\left(X_{0}, Y_{0}\right),(9)$ and (10) we get that for each $k \geqslant 1$ there exist complex numbers $t_{1 k}, t_{2 k}, \ldots, t_{s k}$ such that

$$
a\left(\zeta^{k} p+\sum_{r=1}^{k} \zeta^{k-r} \sum_{i=1}^{s} t_{i r} p_{i}\right)=\zeta^{k} p+\sum_{r=1}^{k-1} \zeta^{k-r} \sum_{i=1}^{s} t_{i r} p_{i}+\sum_{i=1}^{s} t_{i k} y_{i}
$$

where the action of $a$ is in $(V, W)$.

Substituting for $y_{i}$ from (9) we get that the right-hand side of (11)

$$
=\zeta^{k} p+\sum_{r=1}^{k-1} \zeta^{k-r} \sum_{i=1}^{s} t_{i r} p_{i}+\sum_{i=1}^{s}\left[t_{i k}\left(\sum_{j=0}^{m_{i}} \gamma_{j i} \zeta^{j}+\sum_{l=2}^{n} \chi_{i i} w_{l}\right)\right] \text {. }
$$

After applying the definition of the $a$ action on the left-hand side of (11) and equating the coefficients of $w_{l}, l=2, \ldots, n$, one gets

$$
\sum_{j=0}^{m} \gamma_{j} \alpha_{j+k, l}+\sum_{r=1}^{k} \sum_{i=1}^{s} t_{i r} \sum_{j=0}^{m_{i}} \gamma_{j i} \alpha_{j+k-r, l}=\sum_{i=1}^{s} t_{i k} \chi_{i^{*}}
$$

This gives

$$
\sum_{i=1}^{s} t_{i k}\left(\chi_{l i}-\sum_{j=0}^{m_{i}} \gamma_{j i} \alpha_{j l}\right)=\sum_{j=0}^{m} \gamma_{j} \alpha_{j+k, l}+\sum_{r=1}^{k-1} \sum_{i=1}^{s} t_{i r} \sum_{j=0}^{m_{i}} \gamma_{j i} \alpha_{j+k-r, l}
$$

For all $k(12)$ yields an $(n-1) \times s$ matrix $A$

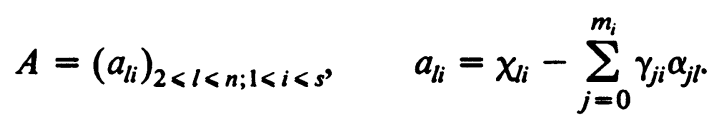

We can now put (12) in the form

$$
A\left[\begin{array}{c}
t_{1 k} \\
\vdots \\
t_{s k}
\end{array}\right]=\left[\begin{array}{cccc}
\sum_{j=0}^{m} \gamma_{j} \alpha_{j+k, 2} & +\sum_{r=1}^{k-1} & \sum_{i=1}^{s} t_{i r} & \sum_{j=0}^{m_{i}} \gamma_{j i} \alpha_{j+k-r, 2} \\
\vdots & \vdots & \vdots & \\
\sum_{j=0}^{m} \gamma_{j} \alpha_{j+k, n} & +\sum_{r=1}^{k-1} & \sum_{i=1}^{s} t_{i r} & \sum_{j=0}^{m_{i}} \gamma_{j i} \alpha_{j+k-r, n}
\end{array}\right]
$$


where the right-hand side is an $(n-1) \times 1$ matrix. Since $s+1<n-1$, $s<n-2$. So the rank of the matrix $A<s$. Therefore there exists a row of $A$ that is a C-linear combination of the other rows. For simplicity of notation we assume that the row in question is the first row. The same proof works for other rows mutatis mutandis. Let the required complex numbers in such a linear combination be $\pi_{3}, \pi_{4}, \ldots, \pi_{n}$. Since we are assuming the existence of $t_{1 k}, \ldots, t_{s k}$ such that (13) holds, the first row of the right-hand side of (13) is a linear combination of the other rows by means of the same scalars $\pi_{3}, \ldots, \pi_{n}$. The matrix $A$ is independent of $k$, so the same scalars work for $k=1,2,3, \ldots$.

Let $L$ be the set of primes used in the definition of the system operation of ( $V, W)$ excepting the first prime, i.e., 2 , and $K=$ the field extension of $Q_{L}$ in the notation of 1.14 obtained by adjoining to $Q_{L}$ the following:

(i) $\pi_{3}, \pi_{4}, \ldots, \pi_{n}$;

(ii) the coefficients of $p, p_{i}, i=1, \ldots, s$, where $p$ is the polynomial part of $y$ in (10) and $p_{i}$ are the polynomials in (9);

(iii) $\chi_{d i}, l=2, \ldots, n, i=1, \ldots, s$, as in (9);

(iv) $\sqrt{2}, \sqrt{3}, \ldots, \sqrt{m}, m=\operatorname{deg} p$. Recall that $m>\max \left(m_{1}, \ldots, m_{s}\right)$.

The matrix $A$ in (13) is over the field $K$, and the algebraic part $K_{0}$ of $K$ over $Q$ is a finite field extension of $Q_{L}$; hence $K_{0}=Q_{L}(\alpha), \alpha$ a complex number.

We shall prove by induction that

$K$ contains square roots of all positive integers and the $t_{i k}$ 's of (11) for all $k=1,2, \ldots$.

For $k=1$ (13) becomes

$$
A\left[\begin{array}{c}
t_{11} \\
\vdots \\
t_{s 1}
\end{array}\right]=\left[\begin{array}{c}
\sum_{j=0}^{m} \gamma_{j} \alpha_{j+1,2} \\
\vdots \\
\sum_{j=0}^{m} \gamma_{j} \alpha_{j+1, n}
\end{array}\right] .
$$

By the definition of $K$ and the fact that $\sum_{j=0}^{m} \gamma_{j} \alpha_{j+1,2}$ is a $K$-linear combination of the other rows, the right-hand matrix is over $K$. We can solve for $t_{11}, \ldots, t_{s 1}$ in terms of the entries in $A$ and the entries of the matrix on the right-hand side of (15); hence $t_{11}, \ldots, t_{s 1}$ are in $K . \sqrt{2}, \sqrt{3}, \ldots, \sqrt{m}$ are in $K$ by definition. We now assume that $t_{11}, \ldots, t_{s 1}$, $t_{12}, \ldots, t_{s 2}, \ldots, t_{1 k-1}, \ldots, t_{s k-1}$ and $\sqrt{m^{\prime}+j-1}, j=1,2, \ldots, k-1$, are contained in $K$ for all $1 \leqslant m^{\prime}<m$. Reverting to (13), we know that the first row of the matrix on the right-hand side is a $K$-linear combination of the 
other rows. By the induction hypothesis this $K$-linear combination is in $K$.

Therefore

$$
\sum_{j=0}^{m} \gamma_{j} \alpha_{j+k, 2}+\sum_{r=1}^{k-1} \sum_{i=1}^{s} t_{i r} \sum_{j=0}^{m_{i}} \gamma_{j i} \alpha_{j+k-r, 2} \in K
$$

Since $m_{i}<m$ for all $i=1, \ldots, s$ the second term of the last expression is in $K$ by the induction hypothesis. Therefore

$$
\sum_{j=0}^{m} \gamma_{j} \alpha_{j+k, 2} \in K, \quad \sum_{j=0}^{m-1} \gamma_{j} \alpha_{j+k, 2} \in K
$$

by the induction hypothesis; hence $\gamma_{m} \alpha_{m+k, 2} \in K$. Since $\gamma_{m} \neq 0$ this implies that $\alpha_{m+k, 2}=\sqrt{m+k} \in K$. Therefore the entries of $A$ and the matrix on the right-hand side of (13) are in $K$. As in the case $k=1$, we conclude that $t_{1 k}, \ldots, t_{s k}$ are in $K$. By induction we have proved (14). Therefore $K_{0}=$ $Q_{L}(\alpha)$, the algebraic part of $K$ contains $Q_{\{2\}}$ contradicting Lemma 1.14. So the assumption that $(V, W)$ has an infinite-dimensional pure subsystem of rank $s+1$ is false. By induction, $(V, W)$ does not have an infinite-dimensional pure subsystem. Hence $(V, W)$ is purely simple by an argument identical to the proof that $(X, Y)$ infinite-dimensional of rank $s+1$ is purely simple. This proves the theorem for $n \geqslant 2$. However, torsion-free rank 1 systems are purely simple by $[4, \mathrm{p} .433]$.

Remark. Also in [4] is the case $n=2$ of Theorem 3.1.

4. A bound on the rank of purely simple systems. We shall first prove some easy lemmas.

LEMMA 4.1. Let $(U, Z)$ be a torsion-free system and $(V, W)$ a torsion-closed subsystem of $(U, Z)$. If $(X, Y)$ is a rank 1 subsystem of $(U, Z)$ not contained in $(V, W)$ then $(V, W) \cap(X, Y)=0$. In particular distinct torsion-closed rank 1 subsystems of $(U, Z)$ intersect trivially.

Proof. Suppose $(V, W) \cap(X, Y) \cdot 0$. By torsion-freeness this implies that $W \cap Y \neq 0$. Therefore $(X, Y)=\operatorname{tc}_{(X, Y)}(\varnothing, y), 0 \neq y \in W \cap Y$. But

$$
\operatorname{tc}_{(X, Y)}(\varnothing, y) \subset \operatorname{tc}_{(U, Z)}(\varnothing, y)=\operatorname{tc}_{(V, W)}(\varnothing, y) \subset(V, W) .
$$

The last equality comes from the fact that $(V, W)$ is torsion-closed in $(U, Z)$. So $(X, Y) \subset(V, W)$, contradiction.

LEMMA 4.2. Let $\left\{w_{i}\right\}_{i \in I}$ be a basis with respect to generation of a torsion-free system $(V, W)$. Then $\sum_{i \in I} \mathrm{tc}_{(V, W)}\left(\varnothing, w_{i}\right)$ is a direct sum.

Proof. Denote tc $(V, W)\left(\varnothing, w_{i}\right)$ by $\left(X_{i}, Y_{i}\right)$. Suppose

$$
\left(X_{l}, Y_{l}\right) \cap \sum_{k \in K}\left(X_{k}, Y_{k}\right) \neq 0
$$


where $K$ is a finite subset of $I$ not containing $l$. Then as in the last lemma $Y_{l} \cap \Sigma_{k \in K} Y_{k} \neq 0$. Let $0 \neq y \in Y_{l} \cap \Sigma_{k \in K} Y_{k}$. Since $\left(X_{l}, Y_{l}\right)$ is of rank 1, it is also equal to

$$
\operatorname{tc}_{\left(X_{l}, Y_{l}\right)}(\varnothing, y) \subset \operatorname{tc}_{(V, W)}\left(\sum_{k \in K}\left(X_{k}, Y_{k}\right)\right) \subset \operatorname{tc}_{(V, W)}\left(\varnothing,\left\{w_{k}\right\}_{k \in K}\right)
$$

so $w_{l} \in \operatorname{tc}_{(v, w)}\left(\varnothing,\left\{w_{k}\right\}_{k \in K}\right)$, contradicting the hypothesis that $\left\{w_{i}\right\}_{i \in I}$ form a basis with respect to generation.

The next lemma is reminiscent of the method of correcting transformations exploited in [1].

Lemma 4.3. Let $(V, W)$ be a system. Suppose $(V, W)=\left(V^{1}, W^{1}\right)+\left(V^{2}\right.$, $\left.W^{2}\right)$ and $(\mu, \nu)$ is a homomorphism from $\left(V^{1}, W^{1}\right)$ to $\left(V^{2}, W^{2}\right)$. Let

$$
V_{\mu}=\left\{v-\mu(v): v \in V^{1}\right\} \text { and } W_{\nu}=\left\{w-\nu(w): w \in W^{1}\right\} \text {. }
$$

Then $(V, W)=\left(V_{\mu}, W_{\nu}\right)+\left(V^{2}, W^{2}\right)$. Moreover $\left(V_{\mu}, W_{\nu}\right)$ is isomorphic to $\left(V^{1}\right.$, $\left.W^{1}\right)$.

PRoof. That $(V, W)=\left(V_{\mu}, W_{\nu}\right)+\left(V^{2}, W^{2}\right)$ is straightforward to check. The map $\left(1_{V^{1}}-\mu, 1_{W^{1}}-v\right)$, where $1_{V}$ denotes the identity map, is an isomorphism between $\left(V^{1}, W^{1}\right)$ and $\left(V_{\mu}, W_{\nu}\right)$.

REMARK 4.4. If $\left(X^{1}, Y^{1}\right)$ is of type $\mathrm{III}^{m_{1}}$ and $\left(X^{2}, Y^{2}\right)$ is of type $\mathrm{III}^{m_{2}}$ with $m_{2}>m_{1}$ then there is a monomorphism from $\left(X^{1}, Y^{1}\right)$ to $\left(X^{2}, Y^{2}\right)$.

Indeed if $\left(X^{1}, Y^{1}\right)$ and $\left(X^{2}, Y^{2}\right)$ are spanned by the respective chains $\left(\left(x_{1}^{1}, \ldots, x_{m_{1}-1}^{1}\right),\left(y_{1}^{1}, \ldots, y_{m_{1}}^{1}\right)\right),\left(\left(x_{1}^{2}, \ldots, x_{m_{2}-1}^{2}\right),\left(y_{1}^{2}, \ldots, y_{m_{2}}^{2}\right)\right)$,

$$
\begin{aligned}
& \phi: x_{i}^{1} \mapsto x_{i}^{2}, \quad i=1, \ldots, m_{1}-1, \\
& \psi: y_{j}^{1} \mapsto y_{j}^{2}, \quad j=1, \ldots, m_{1},
\end{aligned}
$$

extended linearly to $\left(X^{1}, Y^{1}\right)$ is a monomorphism.

THEOREM 4.5. If $(U, Z)$ is a torsion-free system of rank $r>c$ where $c$ is the cardinality of the continuum then $(U, Z)$ is not purely simple.

Proof. Choose a basis of $(U, Z)$ with respect to generation. Let $\left(U^{1}, Z^{1}\right)$ be the subsystem of $(U, Z)$ generated by all but one element of the above basis. If it has no direct summand of type $\operatorname{III}^{m}$ then it is pure in $(U, Z)$ by $[7$, Theorem 1]. So we may assume that

$$
\left(U^{1}, Z^{1}\right)=\left(X^{0}, Y^{0}\right)+(V, W) \text { where }\left(X^{0}, Y^{0}\right) \text { is of type } \mathrm{III}^{m} \text {. }
$$

(Note that for the rest of the proof any reference to $m$ is to the $m$ in (16).)

Rank of $(V, W)=r>c$ by Theorem 1.5. Let $\left\{w_{i}\right\}_{i \in I}$ be a basis of $(V, W)$ with respect to generation, and $\left(X^{i}, Y^{i}\right)=\mathrm{tc}_{(V, w)}\left(\varnothing, w_{i}\right)=\operatorname{tc}_{(U, Z)}\left(\varnothing, w_{i}\right)$. If any of these $\left(X^{i}, Y^{i}\right)$ is infinite-dimensional, it would be pure in $(U, Z)$ by [4, Theorem 5.2]. Therefore to complete the proof of the theorem we may assume 
that

$$
\left(X^{i}, Y^{i}\right) \text { is of type } \text { III }^{m_{i}} \text { for all } i \in I .
$$

By Lemma 4.2 we get

$$
\left(X^{0}, Y^{0}\right) \dot{+} \sum_{i \in I} \cdot\left(X^{i}, Y^{i}\right) \subset\left(U^{1}, Z^{1}\right) .
$$

Our first goal is to prove

$$
\left(U^{1}, Z^{1}\right) \text { has a subsystem } \Sigma_{j \in J} \cdot\left(U_{j}, Z_{j}\right) \text { where each }
$$
$\left(U_{j}, Z_{j}\right)$ is a direct summand of $\left(U^{1}, Z^{1}\right)$ of type III ${ }^{m}$, and $\operatorname{card}(J)=\operatorname{card}(I)=r$.

Proof of (19). Let

$$
\begin{aligned}
& I_{1}^{0}=\left\{i \in I:\left(X^{i}, Y^{i}\right) \text { is a direct summand of }(V, W)\right\}, \\
& I_{2}^{0}=\left\{i \in I: m_{i} \geqslant m, m_{i} \text { as in (17) }\right\} .
\end{aligned}
$$

If $\operatorname{card}\left(I_{1}^{9}\right)=r,(19)$ follows from $(18)(0)$, and if $\operatorname{card}\left(I_{2}^{9}\right)=r$, it follows from 4.4 and Lemma 4.3 with

$$
\begin{aligned}
(V, W) & =\left(X^{0}, Y^{0}\right) \dot{+} \sum_{i \in I_{2}^{0}} \cdot\left(X^{i}, Y^{i}\right),\left(V^{1}, W^{1}\right)=\left(X^{0}, Y^{0}\right),\left(V^{2}, W^{2}\right) \\
& =\sum_{i \in I_{2}^{0}} \cdot\left(X^{i}, Y^{i}\right) .
\end{aligned}
$$

We may therefore assume that the set

$$
I_{3}^{0}=\left\{i \in I: m_{i}<m \text { and }\left(X^{i}, Y^{i}\right) \text { is not a direct summand of }(V, W)\right\}
$$

has cardinality $r$. By [1, Theorem 6.6], for $i$ in $I_{3}^{0},\left(X^{i}, Y^{i}\right)$ is contained in $\left(X_{1}^{i+1}, Y_{1}^{i+1}\right)+\left(X_{2}^{i+1}, Y_{2}^{i+1}\right)$, each of type III $^{m_{i}+1}$. The set

$$
\left\{w_{1}^{i}, w_{2}^{i}: w_{k}^{i} \text { is a generator of }\left(X_{k}^{i+1}, Y_{k}^{i+1}\right), k=1,2 ; i \in I_{3}^{0}\right\}
$$

must have $r$ independent elements with respect to generation in $(V, W)$ because the sum of the systems each pair generates contains $r$ elements independent with respect to generation in $(V, W)$, since $\operatorname{card}\left(I_{3}^{0}\right)=r$. So by 4.2, there is a subset $I_{1} \subset I_{3}^{0}, \operatorname{card}\left(I_{1}\right)=r$ and $\Sigma_{i \in I_{1}} \cdot\left(X_{k}^{i+1}, Y_{k}^{i+1}\right) \subset(V$, $W), k=1$ or 2 . Hence

$$
\left(X_{0}, Y_{0}\right)+\sum_{i \in I_{1}} \cdot\left(X_{k}^{i+1}, Y_{k}^{i+1}\right) \subset\left(U^{1}, Z^{1}\right)
$$

Repeat the same argument on $I_{1}$ as was carried out on $I$ to get $I_{1}^{1}, I_{2}^{1}, I_{3}^{1}$ in place of $I_{1}^{0}, I_{2}^{0} I_{3}^{0}$ and if necessary a set $I_{2}$ of cardinality $r$ and 
$(18)(2)$

$$
\left(X_{0}, Y_{0}\right)+\sum_{i \in I_{2}} \cdot\left(X_{k}^{i+2}, Y_{k}^{i+2}\right) \subset\left(U^{1}, Z^{1}\right),
$$

where $\left(X_{k}^{i+2}, Y_{k}^{i+2}\right)$ is of type $\mathrm{III}^{m_{i}+2}$.

Since $m$ is finite, for some integer $n$, we must get $I_{n} \subset I_{3}^{n-1}$ such that one of the sets $I_{1}^{n}=\left\{i \in I_{n}:\left(X_{k}^{i+n}, Y_{k}^{i+n}\right)\right.$ is a direct summand of $\left.(V, W)\right\}$, $I_{2}^{n}=\left\{i \in I_{n}: m_{i}+n>m\right\}$ has cardinality $r$ and

$(18)(n)$

$$
\left(X_{0}, Y_{0}\right)+\sum_{i \in I_{n}} \cdot\left(X_{k}^{i+n}, Y_{k}^{i+n}\right) \subset\left(U^{1}, Z^{1}\right) .
$$

If $\operatorname{card}\left(I_{1}^{n}\right)=r$, (19) follows from (18) $(n)$ and if $\operatorname{card}\left(I_{2}^{n}\right)=r$ it follows from 4.4 and Lemma 4.3. We have an exact sequence of systems

$$
0 \rightarrow\left(U^{1}, Z^{1}\right) \rightarrow(U, Z) \rightarrow\left(V^{2}, W^{2}\right) \rightarrow 0 .
$$

By the choice of $\left(U^{1}, Z^{1}\right),\left(V^{2}, W^{2}\right)$ is of rank 1. By Lemma 1.9 we may assume that

$$
U=U^{1} \oplus V^{2}, \quad Z=Z^{1} \oplus W^{2},
$$

and that the system operation in $(U, Z)$ is given by

$$
e \cdot\left(u_{1}, v_{2}\right)=\left(e u_{1}+e * v_{2}, e v_{2}\right)
$$

where * is a bilinear map from $\mathbf{C}^{2} \times V^{2}$ to $Z^{1}$. Let $\cup_{j \in J} \cup_{k, \in K,}\left\{z_{k_{j}}\right\}, K_{j}$ a finite set, be a basis of $\Sigma_{j \in J} \cdot Z_{j}$, the range space of the system $\Sigma_{j \in J} \cdot\left(U_{j}, Z_{j}\right)$ of (19).

The cardinality of this basis is $r$. We extend it to a basis, $B$, of $Z^{1}$.

Define a map $f: \mathbf{C} \times V^{2} \rightarrow$ finite subsets of $Z^{1}$ as follows:

$$
\begin{aligned}
f\left(e, v_{2}\right) & =\left\{z_{1}, \ldots, z_{n}\right\} \subset Z^{1} \text { if } \\
e * v_{2} & =\sum_{l=1}^{n} \alpha_{l} z_{l}, z_{1}, \ldots, z_{n} \text { distinct elements of } B
\end{aligned}
$$

and $\alpha_{l} \neq 0$ for all $l=1, \ldots, n$.

The cardinality of $\mathbf{C}^{2} \times V^{2}$ is $c$. Therefore there exists $Z_{1} \in\left\{Z_{j}\right\}_{j \in J}$, the set of range spaces in (19) such that for $z \in Z_{1}$ and $z=\sum_{j=1}^{t} \alpha_{j} z_{j}, \alpha_{j} \neq 0$ for $j=1, \ldots, t, z_{j} \in B$, then $\left\{z_{j}\right\}_{j=1}^{t} \cap f\left(e, v_{2}\right)=\varnothing$ for all $\left(e, v_{2}\right) \in \mathrm{C}^{2} \times V^{2}$. This follows from the fact that excluding the zero element, the $Z_{j}$ 's are mutually disjoint and there are $r$ of them. The theorem will be proved if we show that $\left(U_{1}, Z_{1}\right)$ is a direct summand in $(U, Z)$ because a direct summand of a system is a pure subsystem. By (19) we have $\left(U^{1}, Z^{1}\right)=\left(U_{1}, Z_{1}\right)+\left(V^{1}\right.$, $\left.W^{1}\right)$ for some system $\left(V^{1}, W^{1}\right) \subset\left(U^{1}, Z^{1}\right)$. Therefore

$$
U=\left(U_{1}+V^{1}\right) \oplus V^{2}, \quad Z=\left(Z_{1}+W^{1}\right) \oplus W^{2} .
$$

It is enough to show that $e\left(v^{1}, v^{2}\right) \in W^{1} \oplus W^{2}$ for all $e \in \mathbf{C}^{2}$ and $\left(v^{1}\right.$, $\left.v^{2}\right) \in V^{1} \oplus V^{2}$ for in that case $(U, Z)$ would be equal to

$$
\left(U_{1}, Z_{1}\right) \dot{+}\left(V^{1} \oplus V^{2}, \mathbf{C}^{2}\left(V^{1} \oplus V^{2}\right)\right) \dot{+}\left(0, W^{3}\right)
$$


where $W^{3}$ is any complement in $Z$ of $Z_{1}+\mathrm{C}^{2}\left(V^{1} \oplus V^{2}\right)$. Let $\left(v_{1}, v_{2}\right) \in V^{1}$ $\oplus V^{2}$.

$$
e\left(v_{1}, v_{2}\right)=\left(e v_{1}+e * v_{2}, e v_{2}\right) \text {. }
$$

$e v_{1} \in W^{1}$ because $\left(V^{1}, W^{1}\right)$ is a subsystem of $\left(U^{1}, Z^{1}\right)$, and $e v_{2} \in W^{2}$. Let $e * v_{2}=\sum_{l=1}^{n} \alpha_{l} z_{l}, z_{l}$ distinct elements of $B$ and $\alpha_{l} \neq 0$ for all $l=1, \ldots, n$.

$$
f\left(e, v_{2}\right)=\left\{z_{1}, \ldots, z_{n}\right\} \text {. }
$$

By the choice of $Z_{1}, z_{1}, \ldots, z_{n}$ must all lie in $W^{1}+W^{2}$, hence $e * v_{2} \in W^{1}$ $\oplus W^{2}$. Therefore $e\left(v_{1}, v_{2}\right)$ is in $W^{1} \oplus W^{2}$ as required. This completes the proof of Theorem 4.5.

I do not know if there exists a purely simple system of any infinite rank. However we obtain some information from 4.7 from which we could derive 4.5 as a corollary.

Definition 4.6. A nonzero subsystem $(X, Y) \subset(V, W)$ is said to be of corank 1 if $(V, W) /(X, Y)$ is of rank 1 .

TheOREM 4.7. Let $(V, W)$ be a purely simple torsion-free system. Then $\operatorname{dim}((V, W) /(X, Y))>\operatorname{rank}(V, W)$ for all torsion-closed subsystems $(X$, $Y) \subset(V, W)$ of corank 1 .

Proof. If $\operatorname{rank}(V, W)=1$, the conclusion of the theorem is vacuously satisfied. Let $2<\operatorname{rank}(V, W)<\infty$. Then the hypothesis and Theorem 2.1 exclude finite-dimensional systems. By Lemma 1.12, any torsion-closed subsystem $(X, Y) \subset(V, W)$ of corank 1 is a system of type $\bigoplus_{k \in K} \mathrm{III}^{m_{k}}$, $\operatorname{card}(K)<\infty$. So $\operatorname{dim}(V, W) /(X, Y)$ is infinite. So let $\operatorname{rank}(V, W)=r$, an infinite cardinal. We observe that in the notation of the proof of 4.5 ,

$$
f\left(e, v_{2}\right) \cap\left\{z_{j}\right\}_{j=1}^{t}=\varnothing
$$

for all $\left(e, v_{2}\right) \in \mathbf{C}^{2} \times V^{2}$ if and only if $(f(a, v) \cup f(b, v)) \cap\left\{z_{j}\right\}_{j=1}^{t}=\varnothing$ for all $v$ in a basis of $V^{2}$ and $(a, b)$ a basis of $C^{2}$. So if $\operatorname{dim}((V, W) /(X, Y))$ is less than $\operatorname{rank}(V, W)$ we proceed as in 4.5 to get a contradiction to the hypothesis that $(V, W)$ is purely simple.

\section{REFERENCES}

1. N. Aronszajn and U. Fixman, Algebraic spectral problems, Studia Math. 30 (1968), 273-338. MR 39 \# 1468.

2. I. N. Bernštein, I. M. Gel'fand, and V. A. Ponomarev, Coexeter functors and Gabriel's theorem, Uspehi Mat. Nauk 28 (1973), 19-33 = Russian Math. Surveys 28 (1973), 17-32.

3. V. Dlab and C. M. Ringel, Representations of graphs and algebras, Carleton Mathematical Lecture Notes, no. 8, Carleton Univ., Ottawa, Ont., August 1974.

4. U. Fixman, On algebraic equivalence between pairs of linear transformations, Trans. Amer. Math. Soc. 113 (1964), 424-453. MR 30 \#98.

5. U. Fixman and F. Okoh, Extensions of pairs of linear transformations between infinitedimensional vector spaces, Linear Algebra and Appl.(to appear). 
6. U. Fixman and N. Sankaran, The fundamental functors for pairs of linear transformations (to appear).

7. U. Fixman and F. A. Zorzitto, A purely criterion for pairs of linear transformations, Canad. J. Math. 26 (1974), 734-745. MR 50 \#5540.

8. L. Fuchs, Infinite abelian groups, Vols. I, II, Academic Press, New York and London, 1970. MR 41 \#333.

9. P. Gabriel, Unzerlegbare Darstellungen. I, Manuscripta Math. 6 (1972), 71-103. MR 48 $\# 11212$.

10. Indecomposable representations. II, Symposia Mathematica, Vol. XI (INDAM, Rome, 1971), Academic Press, London and New York, 1973, pp. 81-104. MR 49 \#.5132.

11. F. Okoh, Torsion-free modules over a non-commutative hereditary ring, $\mathrm{Ph}$. D. thesis, Queen's Univ., Kingston, Ontario, 1975.

12. C. M. Ringel, Unions of indecomposable modules, Comm. Algebra 3 (1975), 1121-1144.

13. H. D. Ursell, The degrees of radical extensions, Canad. Math. Bull. 17 (1974), no. 4, 615-617.

14. C. P. Walker, Relative homological algebra and Abelian groups, Illinois J. Math. 10 (1966), 186-209. MR 32 \# 7624.

Department of Mathematics, Queen's University, Kingston, Ontario, Canada K7L 3N6

Current address: Department of Mathematics, University of Nigeria, Nsukka, Nigeria 